\title{
Safety aspects of probiotic products
}

\author{
By Philippe Marteau
}

\begin{abstract}
Probiotics (i.e. ingested living microorganisms) may theoretically be responsible for four types of side effects: systemic infections, deleterious metabolic activities, excessive immune stimulation in susceptible individuals, and gene transfer. Very few cases of adverse events have been reported in humans consuming probiotics, and these are summarized in this paper. I also briefly summarize the three approaches which can be used to assess the safety of a probiotic strain: studies on the intrinsic properties of the strain, studies on its pharmacokinetics, and studies searching for interactions between the strain and the host.
\end{abstract}

Key words: Bifidobacterium, Lactobacillus, probiotics, safety, side effects

\section{Introduction}

Probiotics are commonly defined as viable microorganisms (yeast or bacteria) that exhibit a beneficial effect on the health of the host when they are ingested. They are used in drug formulations but also in foods, especially in fermented dairy products. The use of lactic acid bacteria (LAB) in foods has a long history. There is a growing interest in the development of new and more active strains, and the question of their safety is raised $(1,2)$. We discuss here which adverse effects might theoretically be induced by probiotics, which of them have been documented in literature, and briefly summarize how the safety of probiotic products can be studied.

What are the adverse events which may theoretically be induced by probiotic products and which have been observed?

Probiotics may theoretically be responsible for four types of side effects: systemic infections, deleterious metabolic activities, excessive immune stimulation in susceptible individuals, and gene transfer.

\section{Infections}

Probiotic microorganisms are not selected among pathogens, and the theoretical risk of infections is very low. Rare cases of local or systemic infections including septicemia and endocarditis due to lactobacilli, bifidobacteria or other LAB have been reported $(3,4)$. Most Lactobacillus strains isolated from clinical cases belong to the species L. rhamnosus, L. casei or paracasei, and L. plantarum. Enterococcus faecium and E. faecalis are more frequently involved in clinical infections, and there is concern over the emergence of vancomycin-resistant strains (5). In most cases of infection, the organism appeared to have come from the patient's own microflora. However, in a few cases, the recent use of probiotics by the subject was mentioned as a potential cause. Thirteen cases of fungemia have been reported in humans treated with the probiotic Saccharomyces boulardii (6), and two case of infection have been traced back to food-borne Lactobacillus rhamnosus $(7,8)$. Hennequin et al. (6)

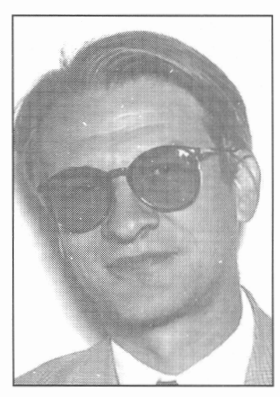

Philippe Marteau, $\mathrm{MD}, \mathrm{PhD}$, Gastroenterology Department, European Hospital Georges Pompidou \& Paris V University, Paris, France.

Address: Service d'Hépato-Gastroentérologie, Hôpital Européen Georges Pompidou, 20 rue Leblanc,F75908 Paris CEDEX 15 France

E-mail: philippe.marteau@egp.ap-hop-paris.fr

The article is based on a lecture presented at the symposium "Probiotics and prebiotics - scientific evidence in humans as a basis for health claims", 14-15 November 2000, arranged by SNF Swedish Nutrition Foundation. reported four cases of fungemia due to $S$. boulardii and discussed literature data. All thirteen subjects who had fungemia had an indwelling vascular catheter. Contamination of the air, environmental surfaces, and hands of the nurses following the opening of the probiotic packets strongly suggested that catheter contamination was the source of infection.

It is now therefore recommended for hospitalized patients that packets or capsules of $S$. boulardii (and in my opinion of any probiotic) should be opened (only whenever needed) with gloves and outside the patient's room (6).

The case of infection due to L. rhamnosus similar to the GG strain was observed in a 74-year-old woman with non-insulin dependent diabetes (7). She suffered from a liver abscess, associated with a right basal pneumonia and a right-sided pleural empyema. No obvious cause of liver abscess was found, and the hepatic abscess aspirate showed that the microorganism was a $L$. rhamnosus. The woman reported a daily intake of about one-half litre of dairy drinks containing L. rhamnosus GG during the 4 months before her symptom. The clinical strain was compared with different $L$. rhamnosus strains, and appeared to be indistinguishable from the GG strain. Another case of infection due to L. rhamnosus was recently published (8). It concerned a 67-year-old man with a mild mitral valve regurgitation, and who had carious teeth removed and who received as prophylaxis $3 \mathrm{~g}$ amoxicillin $1 \mathrm{~h}$ before the procedure. This man was used to consuming probiotic capsules containing a mixture of $L$. rhamnosus + L. acidophilus $+S$. faecalis and as he had found them too large to swallow, "he was in the habit of emptying their contents into his mouth and chewing them with milk". A few days after the dental extraction, he suffered from an endocarditis, and $L$. rhamnosus was isolated from several blood cultures. Further analysis showed that one of the organisms cultured from the probiotic capsule was indistinguishable from that isolated from the blood (cultural appearance, API50, sensitivity pattern, pyrolysis mass spectrometry) (8).

In order to assess the potential of probiotic lactobacilli to cause serious infections, Saxelin et al. $(9,10)$ studied the prevalence of bacteriemia due to Lactobacillus species in Southern Finland during a 4-year period and a 6-year period, and compared the characteristics of the blood culture isolates and of dairy strains. In their first study, lactobacilli were identified in eight out of 3,317 blood culture isolates and none of the isolates corresponded to a dairy strain. In the second study, 5,912 blood cultures were analysed, and none of the 12 lactobacilli isolated was identical to any of the commercial Lactobacillus strains (10).

To summarize, the risk of infection is not nil but extremely low. Nearly all patients with infections due to probiotic micro- 
organisms or to microorganisms close to probiotics have had underlying conditions which predisposed them to infection, particularly abnormal heart valves in the case of endocarditis, and the presence of a catheter in cases of septicemia. Other risk factors for opportunists such as extremes of age, pregnancy, immunodeficiency or digestive lesions have not been identified as risk factors for probiotic infections.

\section{Metabolic effects}

If one admits that probiotics can vehiculate or promote metabolic activities in the gut that may have positive effects for health, one may also admit that they may induce other metabolic activities which may be detrimental to the host.

During bacterial colonization of the small bowel, the microorganisms present in the small bowel in high numbers can induce diarrhoea and intestinal lesions, especially through deconjugation and dehydroxylation of bile salts (11). After ingestion of some probiotics, the concentrations of microorganisms transiting through the small bowel reach the same order of magnitude as that observed during small bowel bacterial overgrowth (11). In a study performed in healthy humans with a terminal ileostomy, we demonstrated that L. acidophilus and Bifidobacterium sp. ingested as fermented dairy products could transform conjugated primary bile salts into toxic free secondary bile salts in the small bowel (11). As this biological effect was only minimal, although statistically significant, it should not be considered as a dangerous side effect of the tested product. However, this study drew attention to the potential risk of excessive deconjugation or dehydroxylation of bile salts in the small bowel by probiotics.

Excessive degradation of the intestinal mucus layer by probiotics may theoretically be detrimental. Some endogenous bacteria including lactobacilli and some strains of bifidobacteria have the ability to degrade mucus. Ruseler-van Embden et al. studied the mucus-degrading properties of three probiotic strains contained in fermented milks (L. acidophilus, Bifidobacterium sp, L. rhamnosus $\mathrm{GG}$ ). No mucus degradation was observed in vitro nor in gnotobiotic rats monoassociated with the test strains (12).

Australian researchers have reported that lactobacilli isolated from cases of infective endocarditis produce enzymes that may enable the breakdown of human glycoproteins, and the synthesis and lysis of fibrin clots. These characteristics aid the colonization and survival of bacteria infecting an endocarditis vegetation (13). However, it remains unknown to date whether they significantly enhance the infectious risk to a relevant extent, and whether they should be considered undesirable in probiotic strains.

\section{Immunological adverse events}

When administered parenterally, bacterial cell wall components such as peptide-glycan-polysaccharides from different grampositive bacteria including lactobacilli can induce side effects such as fever, arthritis or auto-immune diseases (14). These side effects are mediated by cytokines, and it is now well demonstrated that cytokine secretion is elicited by some probiotics (14). This property varies between strains (15). Oral administration of high doses of LAB did not induce immunological side effects in mice (16). However, a systemic uptake of cell wall polymers from the intestinal lumen, hence the immunological side effects, has been observed in rats with colonic injury (17), and during small bowel bacterial overgrowth (14). To our knowledge, no immunological side effect of a probiotic has been reported in man, except one case of auto-immune hepatitis which might have been enhanced by ingestion of large doses of yoghurt (18). Potential enhancement of other auto-immune diseases by probiotic consumption should be studied.

\section{Gene transfer}

Some antibiotic resistance genes, especially those encoded by plasmids, can be transferred between microorganisms. This property raises the question whether resistance genes can be transferred by probiotics to the endogenous flora or to pathogens. McConnel et al. demonstrated that the plasmid pAMß1, which codes for macrolide resistance, could be transferred from Lactobacillus reuteri to E. faecium, and E. faecalis in the mouse gastrointestinal tract (19). The risk of gene transfer depends on the nature of the genetic material to be transferred (plasmids, transposons, etc.), on the nature of the donor and recipient strains, on their concentrations and contacts, and on selection pressure (especially the presence of antibiotics which can selectively promote the growth of the transconjugants). It is difficult to assess in vivo and in vitro, and it is more difficult to state what probability level of gene transfer is acceptable.

Vancomycin is used more and more often for treating patients with infections caused by gram-positive microorganisms, especially during nosocomial infections due to methicillin-resistant staphylococci. Reports of clinical infections caused by vancomycin-resistant organisms including staphylococci, enterococci, lactobacilli, leuconostocs, and pediococcus have also been more frequent in recent years. Vancomycin resistant Enterococcus faecium infections are especially dangerous (5). The safety, and long-term effects on antibiotic resistance of $E$. faecium strains used as probiotics thus clearly need careful assessment $(2,5)$. The situation is not the same for other LAB, despite the fact that many of them are naturally resistant to vancomycin. Indeed, this natural intrinsic resistance of lactobacilli, leuconostocs and pediococci is chromosomally encoded and not inducible or transferable (20), and these microorganisms are sensitive to many other antibiotics.

\section{How to assess the safety of probiotic products?}

Three approaches can be used to assess the safety of a probiotic strain:

1) studies on the intrinsic properties of the strain,

2) studies on the pharmacokinetics of the strain,

3) studies searching for interactions between

the strain and the host.

\section{Intrinsic properties of probiotic strains}

As discussed above, some enzymatic properties such as excessive deconjugation of bile salts or degradation of mucus might be potentially detrimental and can be studied in vitro.

\section{Pharmacokinetics of probiotics}

The survival of ingested probiotics at different levels of the gastrointestinal tract differs between strains (21). It can be measured in vivo using faecal collection, intestinal intubation techniques or identification of strain on mucosal biopsies. Several in vitro models can help to predict the fate of ingested strains.

\section{Adverse interactions - the probiotic and the host}

Illness related to microbiological agents in food is much more difficult to predict than illness due to chemical agents (22). Experience with pathogenic microorganisms in food has shown that the zero risk does not exist but that quantitative risk assessment is needed. However, the concept of "minimal infective dose" is very difficult to realize because of the large number of microbial and host factors involved and the high potential for individual differences.

Animal studies: Animal models are, unfortunately, of limited value in microbial risk assessment (22). Indeed, there is a high 
variability of response between species which makes extrapolation of results obtained in animals to humans hazardous. However, some models can provide interesting information. For example, gnotoxenic animals can help to predict effects of intrinsic properties of probiotic strains on the host. Experiments performed in animal-susceptible to dieases could also be used to predict the risk of disease exacerbation or amelioration in humans.

Acute toxicity studies were conducted for several strains of probiotics, using the same procedures as acute toxicity studies for chemicals $(23,24)$. No acute toxicity was observed (23). Few studies have shown that gene transfer between microorganisms of the flora can be assessed in animals (19), and the relevance of such models is probably greater than that of in vitro mating experiments which poorly simulate the contacts between bacteria in the gut.

Healthy volunteers and monitoring of safety during clinical trials: A rather large amount of data from short-term clinical trials on healthy volunteers attest the safety of probiotics. In most studies, it is only mentioned that the probiotic did not induce more side effects than the placebo or that its tolerance was excellent. In some studies, the presence (or absence) of gastrointestinal disorders has been especially studied, which seems rational since the first and probably only contact between probiotics and the host occurs in the gastrointestinal tract (2). In a few studies, biological parameters were analysed either because it was thought that the probiotic might have influenced them. For example, we showed that the chronic ingestion of $L$. johnsonii LA1 did not alter the jejunal permeability to proteins in healthy humans (25). In other cases, the safety of probiotics

\section{REFERENCES}

1. Marteau P, de Vrese M, Cellier C, Schrezenmeir J: Protection from gastrointestinal diseases using probiotics. Am J Clin Nutr 2001;73:Suppl 430S-436S

2. Donohue DC, Salminen S, Marteau Ph: Safety of probiotic bacteria. In Lactic acid bacteria - Microbiology and Functional aspects. Salminen S, von Wright A eds, Marcel Dekker, New York, 1998 (2nd ed) pp 369-84.

3. Gasser F: Safety of lactic acid bacteria and their occurrence in human clinical infections. Bull Inst Pasteur 1994;92:45-67.

4. Husni RN, Gordon SM, Washington JA, Longworth DL: Lactobacillus bacteremia and endocarditis: review of 45 cases. Clin Infect Dis 1997;25:1048-55

5. Franz CM, Holzapfel WH, Stiles ME. Enterococci at the crossroads of food safety? Int J Food Microbiol 1999;47:1-24.

6. Hennequin C, Kauffmann-Lacroix C, Jobert A, et al.: Possible role of catheters in Saccharomyces boulardii fungemia. Eur J Clin Microbiol Infect Dis 2000;19:16-20.

7. Rautio M, Jousimies-Somer H, Kauma H, Pietarinen I, Saxelin M, Tynkkynen S, Koskela M: Liver abscess due to a Lactobacillus rhamnosus strain indistinguishable from $L$. rhamnosus strain GG. Clin Infect Dis 1999;28: 1159-60.

8. MacKay A, Taylor M, Kibbler C, Hamilton Miller J: Lactobacillus endocarditis caused by a probiotic microorganism. Clin Microbiol Infect 1999:5:290-2.

9. Saxelin M, Chuang NH, Chassy B, Rautelin H, Mäkelä PH, Salminen S, Gorbach SL: Lactobacilli and bacteriemia in Southern Finland, 1989-1992. Clin Infect Dis 1996;22: 564-6.

10. Saxelin M, Rautelin H, Salminen S, Mäkelä PH: Safety of commercial products with viable Lactobacillus strains. Infect Dis Clin Pract 1996;5:331-5.

11. Marteau P, Gerhardt MF, Myara A, Bouvier E, Trivin F, Rambaud JC: Metabolism of bile salts by alimentary bacteria during transit in human small bowel. Microbiol Ecol Health Dis 1995,8:151-7.

12. Ruseler-van Embden JGH, van Lieshout LMC, Gosselink MJ, Marteau P Inability of Lactobacillus casei strain GG, L. acidophilus, and Bifidobacterium bifidum to degrade intestinal mucus glycoproteins: clearing the way for mucosa-protective therapy. Scand J Gastroenterol 1995;30:675-80.

13. Oakey HJ, Harty DWS, Knox KW: Enzyme production by lactobacilli and the potential link with infective endocarditis. J Appl Bacteriol 1995;78:142-8 was studied following the same rules as for chemicals and using several biological parameters $(23,24)$.

\section{Epidemiology and "pharmacovigilance"}

To date, the long history of use of several probiotics without established risk remains the best proof of their safety.

The studies by Saxelin et al. $(9,10)$ provide an excellent example of an epidemiological surveillance. These authors tried to see whether or not the strains of lactobacilli involved in clinical infections were identical to dairy strains (see above). The value of such studies depends of course on the statistical power, i.e. on the number of cases studied.

Recent consumption of probiotics should be clarified in every new case of severe infection caused by enterococci, lactobacilli, yeast, and the strains involved should be compared with the probiotic ones. The use of $S$. boulardii and $L$. rhamnosus has not been banned, despite the few cases of infections, because many studies have proven their general safety, and even efficacy in man. In other words, the absolute risk is very low and the risk-tobenefit ratio appears favourable.

Available data do not suggest that the risk is higher in immunocompromised subjects.

\section{Conclusions}

The three conclusions which can be made at the present time are:

1) that the zero risk does not exist, and that acceptance of the concept that probiotics may not only have positive effects but potentially also side effects is important

2) that the safety of the current products is excellent

3) that further epidemiological and clinical studies are useful for a proper monitoring of the consumer safety.

14. Marteau P, Cellier C: Immunological effects of biotherapeutic agents. In Biotherapeutic agents and Infectious Diseases. Elmer G, Surawicz C McFarland L (eds). Humana Press Inc, Totowa, New Jersey 1998 :pp121-144.

15. Haller D, Bode C, Hammes WP, Pfeifer AM, Schiffrin EJ, Blum S: Nonpathogenic bacteria elicit a differential cytokine response by intestinal epithelial cell/leucocyte co-cultures. Gut 2000;47:79-87.

16. Perdigon G, De Jorrat MEB, De Petrino SF, De Budeguer MV: Effect of oral administration of Lactobacillus casei on various biological functions of the host. Food Agric Immunol 1991;3:93-102.

17. Sartor RB, Bond TM, Schwab JH: Systemic uptake and intestinal inflammatory effects of luminal bacterial cell wall polymers in rats with acute colonic injury. Infect Immunol 1988;56: 2101-8.

18. Chaiken BH. Yogurt and autoimmune liver disease. Am J Gastroenterol 1994;89:1916-7.

19. McConnel MA, Mercer AA, Tannock GW. Transfer of plasmid pAMß1 between members of the normal microflora inhabiting the murine digestive tract and modification of the plasmid in a Lactobacillus reuteri host. Microb Ecol Health Dis $1991: 4 \cdot 343-55$.

20. Dutka-Malen S, Leclercq R, Coutant V, Duval J, Courvalin P: Phenotypic and genotypic heterogeneity of glycopeptide resistance determinants in gram-positive bacteria. J Bacteriol 1990; 39:1772-8.

21. Marteau P, Vesa T: Pharmacokinetics of probiotics and biotherapeutic agents in humans. Bioscience Microflora 1998;17:1-6

22. Anonymous: A scientific basis for regulations on pathogenic microorganisms in foods. ILSI Europe (International Life Science Institute, Avenue E Mounier, 83 Box 6, B-1200, Brussels.), Summary of a workshop held in May 1993 and organised by the Scientific Committee on Microbiology.

23. Zhou JS, Shu Q, Rutherford KJ, Prasad J, Gopal PK, Gill HS: Acute ora toxicity and bacterial translocation studies on potentially probiotic strains of lactic acid bacteria. Food Chem Toxicol 2000;38:153-61.

24. Wolf BW, Garleb KA, Ataya DG, Casas IA: Safety and tolerance of Lactobacillus reuteri in healthy adult male subjects. Microbiol Ecol Health Dis 1995;8:41-50.

25. Marteau P, Vaerman JP, Dehenin JP, Bord S, Brassart D, Pochart P, Desjeux JF, Rambaud JC: Effect of intrajejunal perfusion and chronic ingestion of Lactobacillus acidophilus strain Lal on serum concentrations and jejunal secretions of immunoglobulins and serum proteins in healthy humans. Gastroenterol Clin Biol 1997;21:293-8. 